\title{
Fault Diagnosis of Squirrel Cage Induction Generator for Wind Turbine Applications Using a Hybrid Deep Neural Network and Decision Tree Approach
}

\author{
Hamid Toshani \\ School of Engineering \\ University of East Anglia (UEA) \\ Norwich, UK \\ H.Toshani@uea.ac.uk
}

\author{
Salman Abdi \\ School of Engineering \\ University of East Anglia (UEA) \\ Norwich, UK \\ S.Abdi-Jalebi@uea.ac.uk
}

\author{
Narges Khadem Hosseini \\ Engineering Department \\ Iran University of Science and \\ Technology \\ Tehran, Iran \\ N_Khadem@elec.iust.ac.ir
}

\author{
Ehsan Abdi \\ Wind Technologies Ltd \\ Cambridge, UK \\ Ehsan.Abdi@windtechnologies.com
}

\begin{abstract}
Phase-to-Phase Fault (PPF) and Phase-to-Ground Fault (PGF) are very likely to occur in the generators of wind turbines. Detecting and classifying these faults are vital to improve reliability and reduce the maintenance cost in wind turbine applications. In this paper, a hybrid approach based on Decision Tree (DT) and Convolution Neural Network (CNN) is proposed to make a high-performance fault diagnosis system to detect and classify PPF and PGF in the squirrel cage induction generators. DT algorithm is used to detect the faulty conditions of the generator using several features that are derived from the electrical signals. CNN model is obtained to predict the kind of faults including PPF and PGF using only faulty data. The accuracy of the proposed fault diagnosis approach is evaluated based on the simulation results for a $1.659 \mathrm{MW}$ wind turbine.
\end{abstract}

Keywords-Wind turbine, squirrel cage induction generator, fault diagnosis, decision tree, deep neural network.

\section{INTRODUCTION}

Wind energy is a clean and sustainable fuel source that is known as one of the fastest-growing energy sources in the world. There is about $743 \mathrm{GW}$ wind power capacity worldwide, where only $92 \mathrm{GW}$ of new capacity is installed in 2020 [1]. It is expected to reach more than $10 \mathrm{GW}$ additions using only offshore wind net capacity up to 2022 [2]. Nowadays, wind turbines are becoming larger, more expensive, and more complicated to generate large amounts of energy. Installing offshore wind turbines costs almost 3.3 million pounds per megawatts [3]. For a relatively large wind turbine, the cost of having reliable performance and maintenance is greatly high.

Meanwhile, the occurrence of different faults for these systems is unavoidable as they work in a relatively harsh environment and are composed of several inter-connecting electro-mechanical components. Accordingly, developing a condition monitoring system to detect and classify faults is indispensable to ensure the reliability, safety, and effectiveness of wind turbines.

The generator with a $12.1 \%$ failure rate has the highest contributions to the overall offshore wind turbine failures [4]. The main faults in a generator occurred in the stator, rotor, bearing, and insulation [5]. The failure rate for the only stator is about $30 \%$ where the most common winding faults in the stator are PPF and PGF [6].

Current signature analysis is a common fault detection approach, where the fault frequency components are analysed based on the spectra of the electrical signals [7]. This technique usually consists of signal conditioning, spectral analysis, and fault detection. The inter-turn short circuit in the stator winding may change the air-gab flux which excites the harmonic frequencies in the stator winding [8]. Wavelet transforms on the stator current signals can be used to determine the fault-related frequency components for a more accurate diagnosis of failure modes [9]. To effectively detect the faults in the rotor, discrete wavelet transform can be used to analyse the fault frequencies of electrical signals [10]. One drawback of the current signature analysis is that the statistical properties of the signature of the faults in electrical signals are changing through time. It is a challenging issue due to the need to design a more complex condition monitoring system [11]. In addition, this method may suffer from weak performance to detect faults with relatively small severity.

As a distinct technique, the instantaneous line currents can be converted into the Parks vectors where they contain a positive sequence component in the three-phase current. These vectors show an elliptic representation in two-dimensional space when a fault occurs in the generator [12]. In this approach, the twice fundamental component of parks vector square can be used as a fault indicator [13]. Park's vectors are not suitable when different faults have close effects on the wind turbine performance. Moreover, its accuracy may decrease if the threephase currents are far from ideal conditions.

Model-based approaches are another method to detect faults in the generator using electrical signals. Sliding mode observer is a technique to estimate the internal states of the generator [14]. By comparing the measured and estimated values of stator currents, the fluctuations duo to the inter-turn faults can be observed. An alternative observer is a neural network that has the capability of estimating nonlinearities in wind turbines regarding uncertainties and faults [15]. Kalman filter is also used to estimate the three-phase currents and the residual signals are used to detect the inter-turn faults as well as fault severity [16]. 
Dependence on mathematical models is accompanied by difficulty as wind turbines consist of various highly coupled components and they are becoming larger to gain more energy.

Feature extraction-based methods are widely used for fault detection in wind turbine applications. They can be used for both stationary and non-stationary signals, have less sensitivity to severity of faults as well ideality of the electrical signals, and have no dependency on the model of the wind turbine. A combination of the wavelet transform and Naïve Bayes classifier is proposed in [17] where the earliest is used for feature extraction and the latter is designed to classify the inter-turn faults. An approach based on wavelet transform and empirical mode decomposition is introduced in [18]. This method determines different modes of the main signal and uses feature extraction to detect the faults. It is suitable for non-stationary signals and has robustness against noise. A challenging issue in these methods is detecting the faults with close impacts on the wind turbine performance. A solution is using deep neural networks as they are capable of reducing and optimally tuning features by themselves. These networks have the robustness to the data variations and do not need data labeling [19]. Deep neural networks are used for fault detection in various wind turbine components like gearbox [20-21], pitch actuator [22], and converter [23].

The main drawbacks of deep neural networks are their complex structure, high computational time, and the requirement for a large amount of data. To overcome these issues, a hybrid and parallel approach based on Decision Tree (DT) and Convolutional Neural Network (CNN) as a common deep learning-based network is proposed in this paper. DT is a powerful approach to easily read and interpret data. It doesn't require normalization and scaling data and has low sensitivity to missing data [24]. The aim of using DT is to detect the faulty condition of the generator using electrical signals. Then, two CNNs are designed to predict the PPF and PGF using only faulty data. Using DT allows using fewer amounts of data for CNN. Indeed, using only faulty data for $\mathrm{CNN}$ simplifies the structure of the neural network and reduces the overall computational time. In order to establish DT, several features are extracted using the main electrical signals for both healthy and faulty conditions.

The rest of this paper is organized as follows: the mathematical model of the wind turbine is described in Section 2. The general structure of the proposed approach is introduced in Section 3. Feature extraction is given in Section 4. The fault detection using $\mathrm{D}$, and fault diagnosis using $\mathrm{CNN}$ along with the simulation results are presented in Sections 5, and 6, respectively. The conclusion is given in Section 7.

\section{MATHEMATICAL MODEL OF WIND TURBINE}

As shown in Fig. 1, the drivetrain mainly includes a gearbox, and a generator, which are connected using a high-speed shaft. The generator terminals are connected to a grid through a $1 \mathrm{Km}$ transmission line.

According to Fig. 2, the gearbox consists of a planetary gear. And two helical gears. The planetary gear includes a carrier, ring, planet, and sun gears. Its turn ratio $N_{p}$ is given below:

$$
N_{p}=\frac{N_{r}}{N_{s}}
$$

where $N_{r}$ and $N_{s}$ are the number of teeth in the ring and sun gears, respectively.

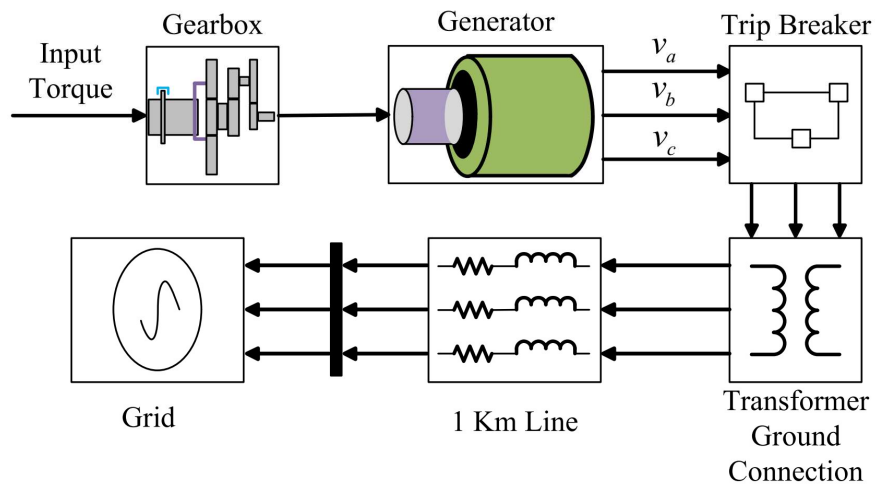

Fig. 1. The main blocks of wind turbine drivetrain

The helical gears include base gear and follower gear.

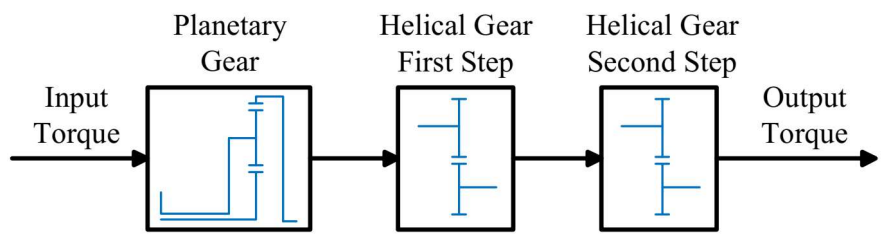

Fig. 2. The main blocks of the gearbox

Their turn ratios are defined below:

$$
N_{h}=\frac{N_{f}}{N_{b}}
$$

where $N_{f}$ and $N_{b}$ are the number of teeth in the follower and base gears, respectively. By applying the mechanical torque $T_{m}$ from the gearbox to the generator, the angular velocity of the rotor $w_{m}$ can be obtained as follows [25]:

$$
\frac{d}{d t} w_{m}=\frac{1}{2 H}\left(T_{e}-F w_{m}-T_{m}\right)
$$

where $T_{e}$ is the electromechanical torque. $H$ and $F$ are the combined rotor and load inertia constant, and viscous friction coefficient, respectively. The electrical part of the asynchronous machine can be represented by a fourth-order state-space model as follows:

$$
\begin{aligned}
& V_{q s}=R_{s} i_{q s}+\frac{d \varphi_{q s}}{d t}+w \varphi_{d s} \\
& V_{d s}=R_{s} i_{d s}+\frac{d \varphi_{d s}}{d t}-w \varphi_{q s} \\
& V_{q r}^{\prime}=R_{r}^{\prime} i_{q r}^{\prime}+\frac{d \varphi_{q r}^{\prime}}{d t}+\left(w-w_{r}\right) \varphi_{q r}^{\prime} \\
& V_{d r}^{\prime}=R_{r}^{\prime} i_{d r}^{\prime}+\frac{d \varphi_{d r}^{\prime}}{d t}-\left(w-w_{r}\right) \varphi_{d r}^{\prime}
\end{aligned}
$$


where $\left(V_{q s}, i_{q s}, \varphi_{q s}\right),\left(V_{d s}, i_{d s}, \varphi_{d s}\right)$, are the q-axis and d-axis stator voltages, currents, and fluxes, respectively. $R_{s}$ and $R_{r}{ }_{r}$ are the stator and rotor resistances, respectively. $w_{r}$ denotes the electrical angular velocity and can be obtained as below:

$$
w_{r}=p \times w_{m}
$$

where $p$ is the number of poles. The reference velocity in the reference frame fixed to the rotor is $w \cdot\left(V_{q r}^{\prime}, i_{q r}^{\prime}, \varphi_{q r}^{\prime}\right)$ and $\left(V_{d r}^{\prime}, i_{d r}^{\prime}, \varphi_{d r}^{\prime}\right)$ are the q-axis and d-axis rotor voltages, currents, and fluxes, respectively. The equations for the rotor and stator fluxes are given below [25]:

$$
\begin{aligned}
& \varphi_{q s}=L_{s} i_{q s}+L_{m} i_{q r}^{\prime} \\
& \varphi_{d s}=L_{s} i_{d s}+L_{m} i_{d r}^{\prime} \\
& \varphi_{q r}^{\prime}=L_{r}^{\prime} i_{q r}^{\prime}+L_{m} i_{q s} \\
& \varphi_{d r}^{\prime}=L_{r}^{\prime} i_{d r}^{\prime}+L_{m} i_{d s}
\end{aligned}
$$

where $L_{m}$ is the magnetizing inductance. $L_{s}$ and $L_{r}^{\prime}$ are the total stator and rotor inductances, respectively. $T_{e}$ can be obtained as follows:

$$
T_{e}=1.5 p\left(\varphi_{d s} i_{q s}-\varphi_{q s} i_{d s}\right)
$$

In this paper, the turn ratio of the gearbox is $1: 84: 3$. The mechanical power, rated power, and rated current of the turbine are $1800 \mathrm{KW}, 1659 \mathrm{KW}$, and $1740 \mathrm{~A}$, respectively. The generator is 4 poles asynchronous induction machine which operates at $50 \mathrm{~Hz}, 1200 \mathrm{rpm}$ rotational speed.

The PPF and PGF are emulated at the terminals of the generators as shown in Fig. 3. The phase resistor $R_{o n}$ is equal to $10^{-6} \Omega$, while the ground resistor $R_{G}$ is chosen $10^{-6} \Omega$ and $10^{6} \Omega$ when it aims to make PPF and PGF, respectively. The circuit breaker has the role of activating PPF and PGF among the operating wind drivetrain.

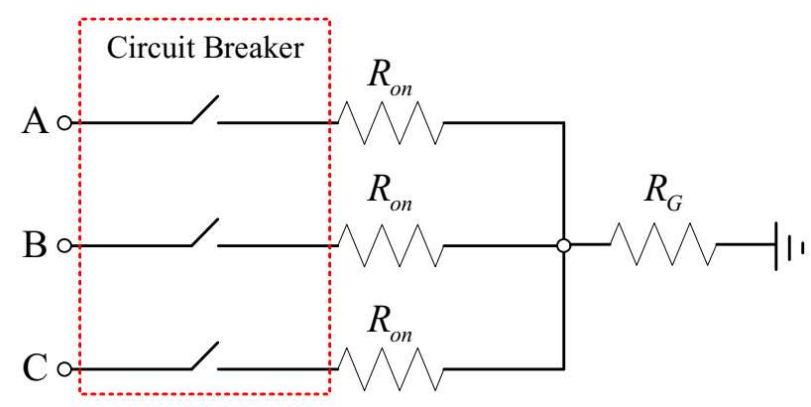

Fig. 3. Emulating PPF and PGF at the terminals of the generator

\section{THE PROPOSED FAULT DIAGNOSIS ALGORITHM}

The general block diagram of the proposed fault diagnosis algorithm is shown in Fig. 4. As it can be seen, the proposed algorithm is composed of three main subsystems: The feature extraction stage, the Fault detection stage, and a Fault classification stage. To detect and classify PPF and PGF in the generator, the three-phase stator currents are gathered for both healthy and faulty conditions at various wind speeds and directions. These signals in addition to their power spectral density are used to extract several features including mean, standard deviation, kurtosis, crest factor, skewness, and shape factor. DT Model is trained using these features for both healthy and faulty conditions. The model is used to carry out the fault detection stage. By using the features for faulty features, a CNN is trained to classify the faults including PPF and PGF.

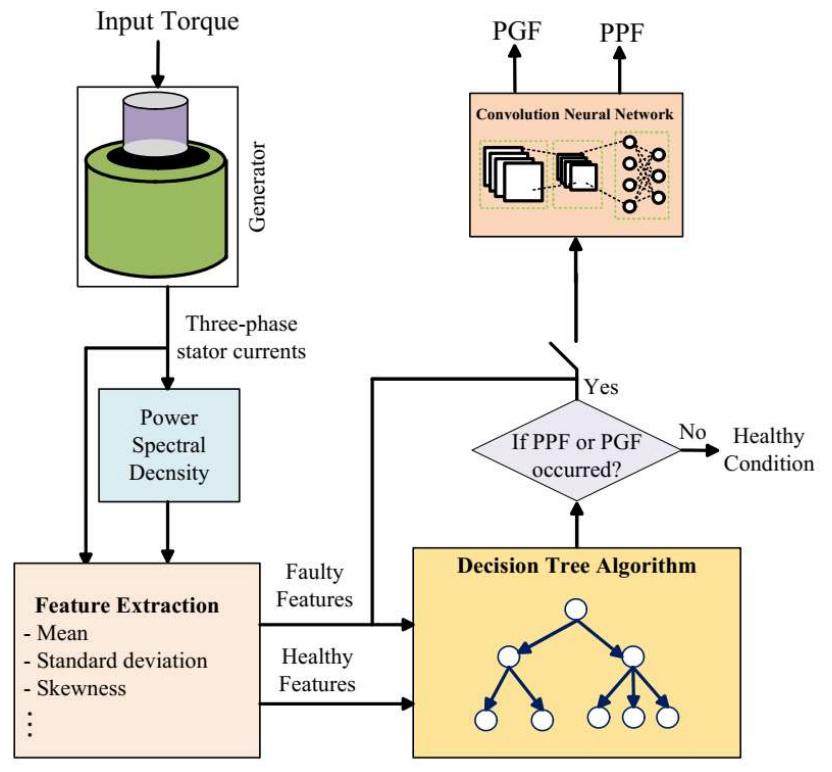

Fig. 4. Block diagram of the proposed fault diagnosis system

\section{FEATURE EXTRACTION}

Feature extraction is an effective approach to reduce the dimension of the original dataset by creating new specifications from the existing ones. Features can be obtained using the timedomain signals, fast Fourier transform, wavelet functions, and Power Spectral Density (PSD). In this paper, three-phase stator currents and their PSD are used to extract the features at various wind profiles. Figure 5 shows the different wind speeds and directions.
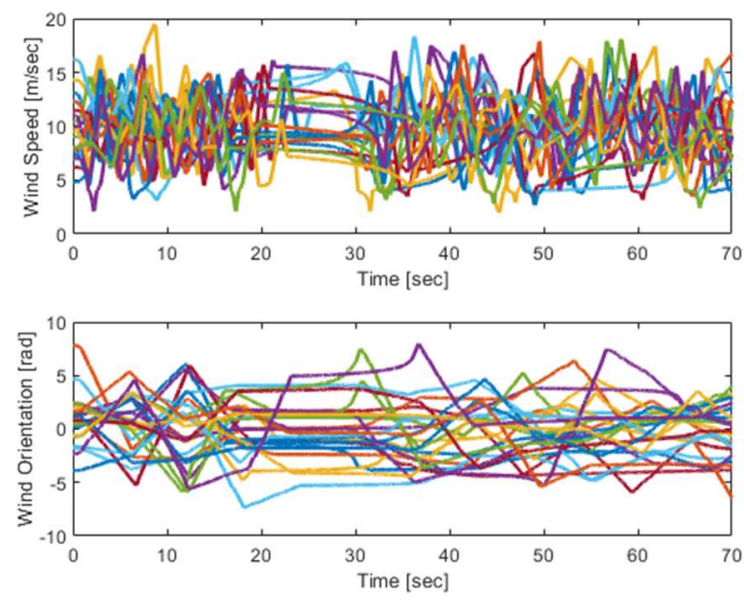

Fig. 5. Various wind speeds and directions

\footnotetext{
Identify applicable funding agency here. If none, delete this text box.
} 
It is supposed that the PPF and PGF occurred at different phases and different periods of time. As an example, the first phase current signal for both healthy and faulty conditions is shown in Fig. 6. The PPF and PGF occurred between $40 \mathrm{sec}$ to $42 \mathrm{sec}$, and between $42 \mathrm{sec}$ and $44 \mathrm{sec}$, respectively.
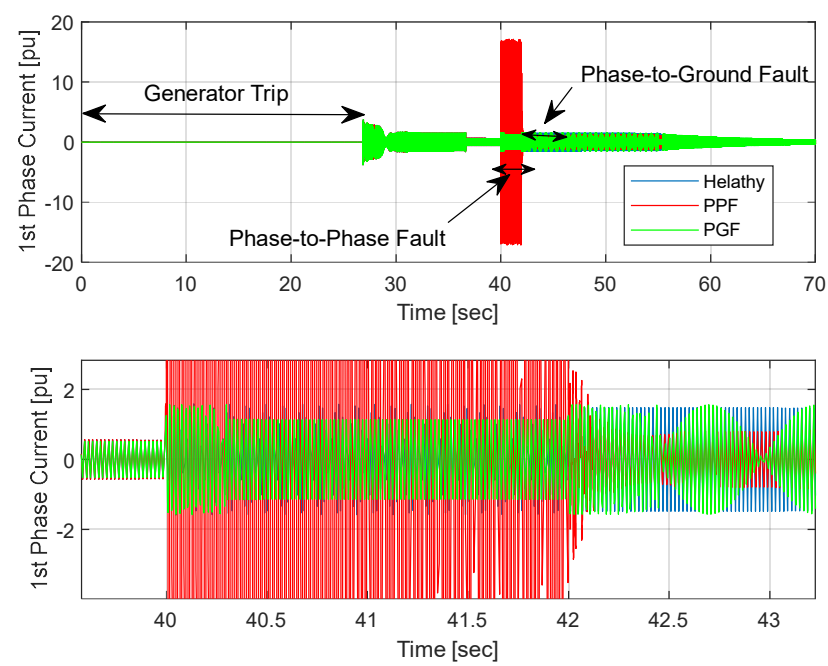

Fig. 6. The healthy and faulty first phase current signals

The PSD of the current signals is also used to extract the features. Figure 7 shows the results of PSD for the healthy and faulty first phase current signals.
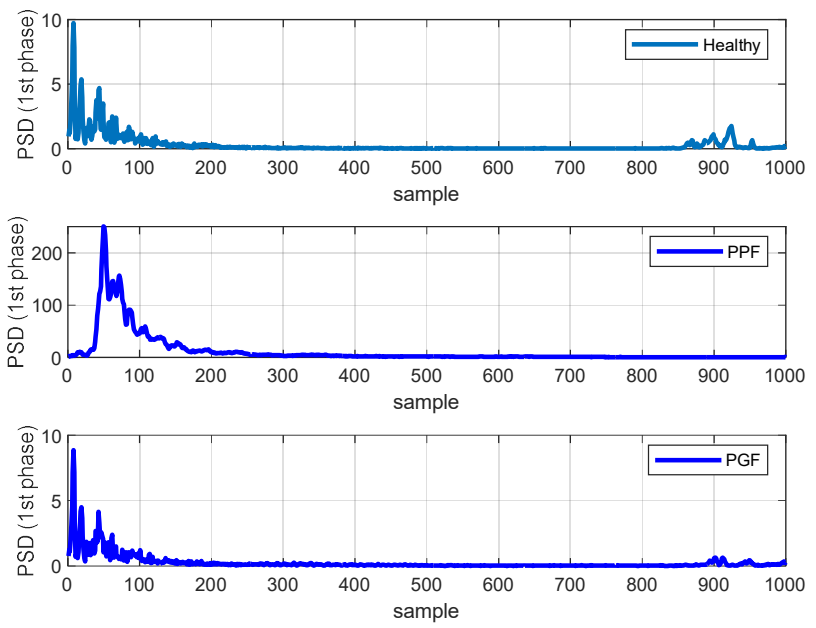

Fig. 7. The PSD of first phase current signal for healthy and faulty conditions

In this paper, mean, standard deviation, skewness, kurtosis, and crest factor are chosen as the features to gather the required data for training DT. Skewness $S_{k}$ is a measure to determine the deviation of a distribution from a normal one and can be described as follows [26]:

$$
S_{k}=\frac{\sum_{i=1}^{N}(x(i)-\bar{x})^{3}}{(N-1) x_{\text {std }}^{3}}
$$

where $\mathbf{x} \in R^{N}$ is the dataset, $N$ is the number of data, $\bar{x}$, and $x_{\text {std }}$ are the mean, and standard deviation, respectively. Kurtosis
$K_{u}$ which is given below is a measure to find how distribution is too peaked or too flat.

$$
K_{u}=\frac{\sum_{i=1}^{N}(x(i)-\bar{x})^{4}}{(N-1) x_{s t d}^{4}}
$$

The crest factor $C_{r}$ is the ratio of peak value to the effective value of a signal. Figure 8 shows the results of skewness for both healthy and faulty conditions. These data along with the results of other features are used as training data for the DT algorithm as well as the CNN.

\section{FAULT DETECTION USING THE DT ALGORITHM}

DT is a hierarchal algorithm that aims to extract the patterns in the dataset for data classification. It is composed of decision rules that are applier the patterns to discriminate the feature space into single class subspaces [27]. The block diagram of the DT algorithm is shown in Fig. 9, where the root node denotes the dataset, the decision node refers to sub-nodes splits to further sub-nodes, and the leaf node is a node that doesn't split. In this paper, the Classification and Regression Tree (CART) algorithm is used to carry out DT. This algorithm uses the following objective function called Gini to measure the reduction in class impurity:
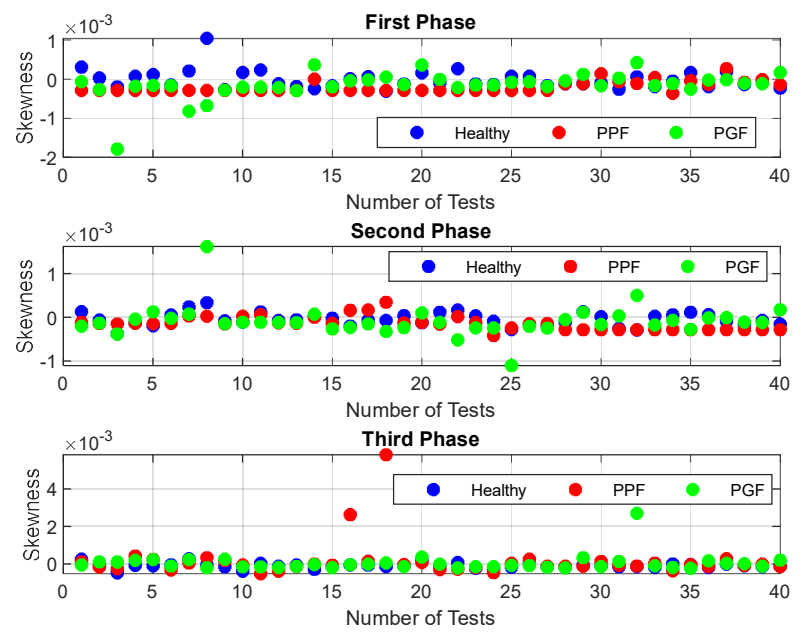

Fig. 8. Skewness values for both healthy and faulty conditions

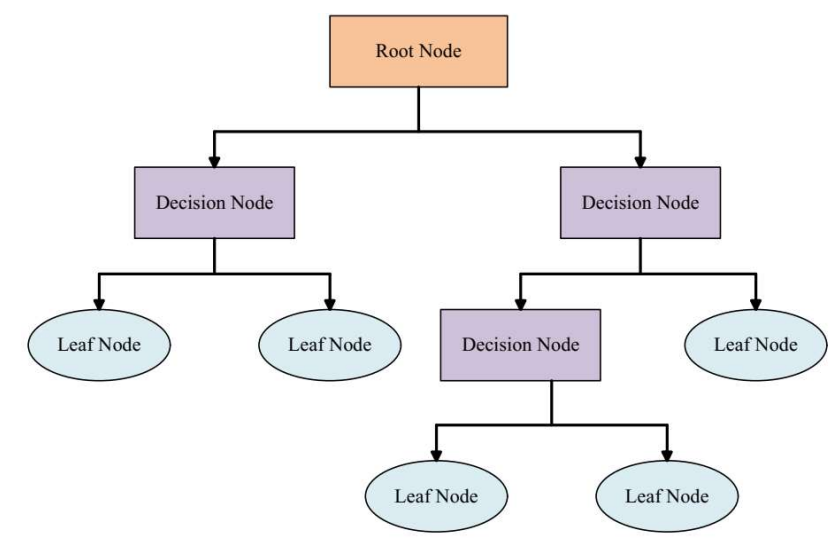

Fig. 9. The block diagram of the DT algorithm [27] 


$$
\text { Gini }=\text { impurity }(\text { Parents })-\sum_{k}\left(p_{k}\right) \text { impurity }\left(\operatorname{Child}_{k}\right)
$$

where the impurity is the probability of incorrectly classifying data and is given below:

$$
\text { impurity }=1-\sum_{j}\left\|p(j) N_{j}(t) / N_{j}\right\|^{2}
$$

where $p(j)$ is the prior probability that the $j$ th sample belongs to a class. $N_{j}$ is the number of samples in class $j$. Parents and Childs refer to decision and leaf nodes.

The dataset for DT is a matrix that includes five features as the rows and 720 data for each column with healthy and faulty labels. The key parameter to achieve the best performance is the number of leaf nodes that corresponds to the minimum objective function. According to Fig. 10, a minimum of five-leaf nodes is required for

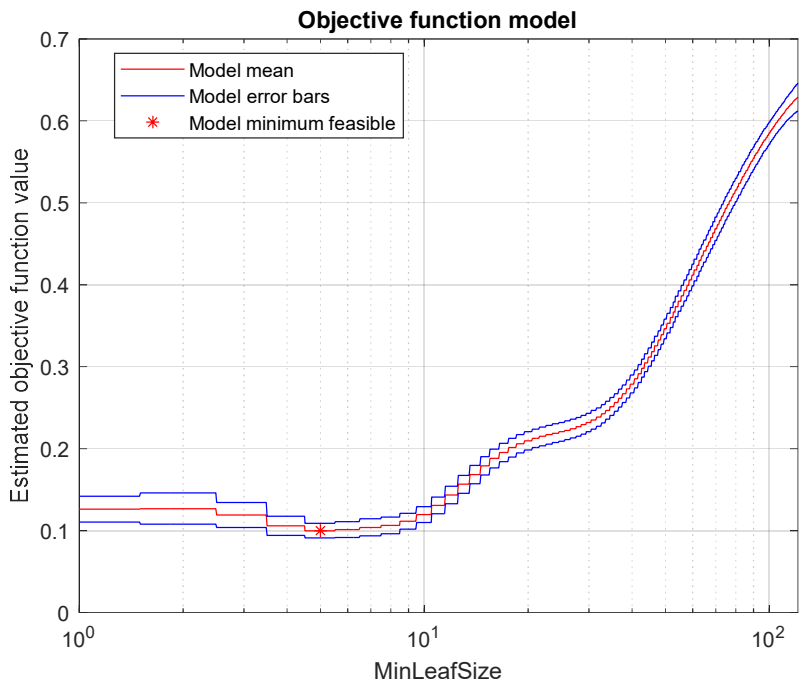

Fig. 10. Obtaining a minimum number of leaf nodes

Figure 11 shows the structure of the DT algorithm which describes the classifying rules for discriminating healthy and faulty conditions. The accuracy of the DT algorithm for classifying healthy and faulty features is $97 \%$.

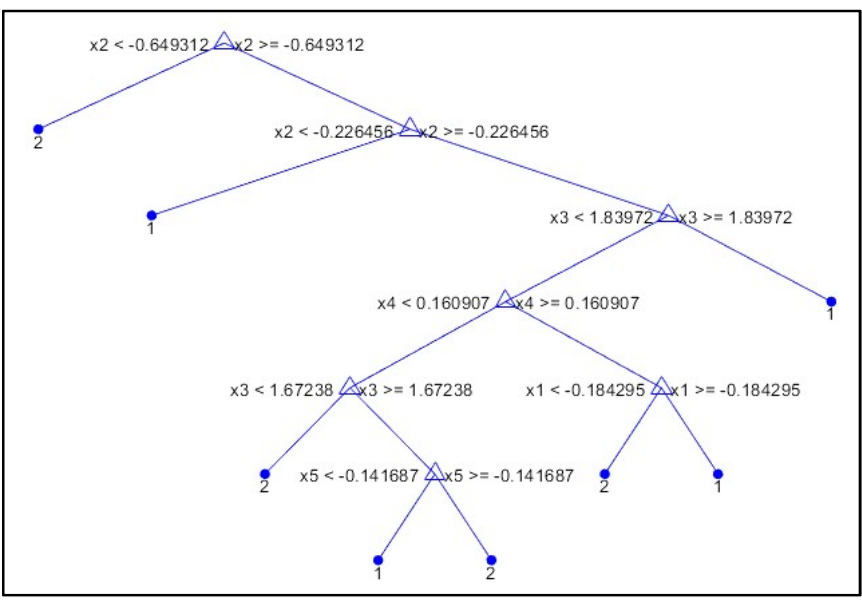

Fig. 11. The classifying rules of the DT algorithm

\section{FAULT DIAGNOSIS USING CNN}

The third stage of the proposed algorithm is classifying PPF and PGF using only faulty features. A CNN model based on the configuration in Fig. 10 is trained where the network consists of a convolution layer, a pooling layer, and a fully connected layer. The local features of the input data are identified and saved as feature maps. The process is carried out using the following convolution [28]:

$$
y_{i j}=h\left(\sum_{r=1}^{F} \sum_{c=1}^{F} w_{r c} x_{(r+i \times s)(c+j \times s)}+b\right)
$$

where $y_{i j}$ is the result of the feature map for each node. $h($.$) is$ the nonlinear activation function, and $b$ is the bias. In addition, $w_{r c}$ is the weight at point $(r, c)$, and $x_{(r+i \times s)(c+j \times s)}$ is an element of input space. $W$ and $H$ are the width and height of input space, respectively. $F$ and $S$ are the width and height of the mapped space.

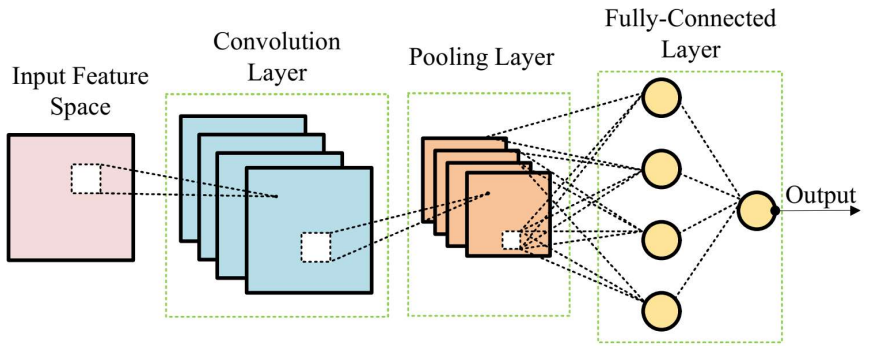

Fig. 12. The block diagram of $\mathrm{CNN}$

The pooling layer has the role of dimension reduction by optimally reducing the number of parameters. The classification process is carried out in the fully connected layer. In order to classify the faults, the features for PPF and PGF are labeled as 2 , and 3, respectively. By considering 80 percent of data for training, and 20 percent of data for testing, the CNN model is obtained. As shown in Fig. 13, the CNN can predict the type of faults in most cases. One of the key parameters to obtain the best performance of $\mathrm{CNN}$ in case of accuracy and root mean square error is the number of hidden units. According to Table 1, the best performance is achieved when 50 hidden units are chosen for $\mathrm{CNN}$.
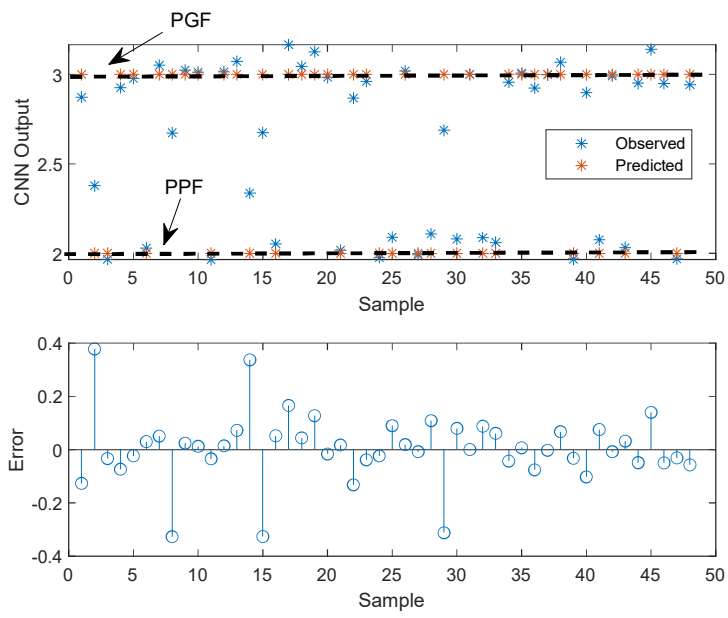

Fig. 13. The results of $\mathrm{CNN}$ for fault diagnosis 
TABLE I. THE ACCURACY OF FAULT DIAGNOSIS STAGE

\begin{tabular}{ccc}
\hline $\begin{array}{c}\text { Number of hidden } \\
\text { units }\end{array}$ & Accuracy (\%) & $\begin{array}{c}\text { Root mean square } \\
\text { error }\end{array}$ \\
\hline 10 & 87.3 & 0.77 \\
\hline 50 & 90.5 & 0.32 \\
\hline 100 & 88.7 & 0.43 \\
\hline
\end{tabular}

\section{CONCLUSION}

In this paper, a hybrid approach based on the DT algorithm and CNN is proposed to detect and classify PPF and PGH in wind drivetrain. A dataset that includes the three-phase current signals and their PSD is provided to extract features. Based on five features including mean, standard deviation, skewness, kurtosis, and crest factor, the input data is applied to the DT. Using the classifying rules of DT, $97.2 \%$ accuracy is obtained in the fault detection stage. By properly training the CNNs using only faulty features, $95.4 \%$ accuracy is achieved in the fault diagnosis stage. In our future works, it aims to consider other kinds of faults in wind drivetrain including air gap eccentricity, rotor imbalance, and broken rotor bar.

\section{REFERENCES}

[1] https://gwec.net/global-wind-report-2021/

[2] https://www.iea.org/reports/renewables-2020/wind

[3] Z. Gao, and X. Liu, "An overview on fault diagnosis, prognosis and resilient control for wind turbine systems," Processes, vol. 9, pp. 1-19, 2021.

[4] J. Carroll, A. McDonald, and D. McMillan, "Failure rate, repair time and unscheduled O\&M cost analysis of offshore wind turbines," Wind Energy, vol. 19, pp. 1107-1119, 2016.

[5] B. Hahn, M. Durstewitz, and K. Rohrig, Reliability of wind turbines, Springer, Berlin, Heidelberg, 2007.

[6] [6] P. V. J. Rodríguez, M. Negrea, and A. Arkkio, "A simplified scheme for induction motor condition monitoring," Mech. Syst. Signal Proc, vol. 22, pp. 1216-1236, 2008.

[7] [7] E. Artigao, S. Koukoura, A. Honrubia-Escribano, J. Carroll, A. McDonald, and E. Gómez-Lázaro, "Current signature and vibration analyses to diagnose an in-service wind turbine drive train," Energies, vol. 11, p. 1-18, 2018

[8] E., Artigao, A., Honrubia-Escribano, and E. Gomez-Lazaro, "Current signature analysis to monitor DFIG wind turbine generators: A case study," Renew. Energy, vol. 116, pp. 5-14, 2018.

[9] E. Artigao, E. Gonzalez, A. Honrubia-Escribano, and E. Gomez-Lazaro, "Analysing Current Signature Data to Diagnose an In-Service Wind Turbine Generator," J. Physics: Conf. Series, IOP Publishing, vol. 1222, pp. 1-11, 2019.

[10] H., Garg, and R. Dahiya, "Current signature analysis and its application in the condition monitoring of wind turbine for rotor faults," Energy Syst., vol. 8, pp. 495-510, 2018.

[11] W. Qiao, and D. Lu, "A survey on wind turbine condition monitoring and fault diagnosis-Part II: Signals and signal processing methods," IEEE Trans. Indust. Elec, vol. 62, pp. 6546-6557, 2015.
[12] E., Artigao, A., Honrubia-Escribano, and E. Gomez-Lazaro, "Condition monitoring of a wind turbine doubly-fed induction generator through current signature analysis," J, Physics: Conference Series, vol. 926, No. 1, p. 1-7, 2017

[13] S. Wei, X. Zhang, Y. Xu, Y. Fu, Z. Ren, and F. Li, "Extended Park's vector method in early inter-turn short circuit fault detection for the stator windings of offshore wind doubly-fed induction generators," IET Gen. Trans. Distr, vol. 14, pp. 3905-3912, 2020.

[14] T. Sellami, H. Berriri, S. Jelassi, A. M. Darcherif, and M. F. Mimouni, "Sliding mode observers-based fault detection and isolation for wind turbine-driven induction generator," Inter. J. Power Elect. Drive Syst, vol. 8, pp. 1345-1358, 2017.

[15] H. Habibi, I. Howard, and S. Simani, "Reliability improvement of wind turbine power generation using model-based fault detection and fault tolerant control: A review," Renewable energy, vol. 135, pp. 877-896, 2019.

[16] H. Sabir, M. Ouassaid, and N. Ngote, "Diagnosis of rotor winding interturn short circuit fault in wind turbine based on DFIG using hybrid TSA/DWT approach," 2018 6th International Renewable and Sustainable Energy Conference (IRSEC), pp. 1-6, 2018.

[17] H. Toshani, S. Abdi, N. Khadem, and E. Abdi, "Wind Turbine Generator Short Circuit Fault Detection Using a hybrid approach of wavelet transform and Naïve Bayes classifier," 2021 IEEE 15th International Conference on Compatibility, Power Electronics and Power Engineering (CPE-POWERENG), pp. 1-7, 2021.

[18] P. Shi, W. Yang, M. Sheng, and M. Wang, ”An enhanced empirical wavelet transform for features extraction from wind turbine condition monitoring signals," Energies, vol. 10, pp. 1-13, 2017.

[19] M. A. Nielsen, Neural networks and deep learning San Francisco, CA: Determination press, 2015.

[20] G. Jiang, H. He, J. Yan, and P. Xie, "Multiscale convolutional neural networks for fault diagnosis of wind turbine gearbox," IEEE Trans. Indust. Elec, vol. 66, pp. 3196-3207, 2018.

[21] L. Wang, Z. Zhang, H. Long, J. Xu, and R. Liu, "Wind turbine gearbox failure identification with deep neural networks," IEEE Trans. Indust. Inform, vol. 13, pp. 1360-1368, 2016.

[22] S. Zare, and M. Ayati, "Simultaneous fault diagnosis of wind turbine using multichannel convolutional neural networks," ISA trans, vol. 108, pp. 230-239, 2021.

[23] C. Xiao, Z. Liu, T. Zhang, and X. Zhang,"'Deep learning method for fault detection of wind turbine converter,"Applied Sciences, vol. 11, pp. 1280, 2021

[24] M. Jena, and S. Dehuri, "Decision tree for classification and regression: A state-of-the art review," Informatica, vol. 44, pp. 405-420, 2020.

[25] P. C. Krause, O. Wasynczuk, S. D. Sudhoff, and S. D. Pekarek, Analysis of electric machinery and drive systems, John Wiley \& Sons, 2013.

[26] A. B. Gholap, and M. D. Jaybhaye, "Vibration Signature Analysis of Two Stage Gearbox using Kurtosis, Skewness and Crest Factor," Manu.Tech. Today, vol. 18, pp. 28-33, 2013.

[27] B. Charbuty, and A. Abdulazeez, "Classification based on decision tree algorithm for machine learning," J. Applied Science and Tech.Trends, vol. 2, pp. 20-28, 2021.

[28] N. Ketkar, "Convolutional neural networks. In Deep Learning with Python, Apress, Berkeley, CA, 2017. 